\title{
Prism adaptation test before strabismus surgery in patients with decompensated esophoria and decompensated microesotropia
}

\author{
Caroline Gietzelt • Julia Fricke $\cdot$ Antje Neugebauer $\cdot$ Andrea Hedergott $(\mathbb{D}$
}

Received: 4 September 2021 / Accepted: 4 January 2022 / Published online: 17 January 2022

(C) The Author(s) 2022

\begin{abstract}
Purpose To evaluate the effect of Prism adaptation test (PAT) on the angle of squint in decompensated esophoria (decEPH) and decompensated microesotropia (decMET).

Methods In this single-center retrospective study we reviewed the medical records of patients with the diagnosis of decEPH or decMET, aged at least 12 years, who were treated by strabismus surgery for the first time. The maximum Angle of squint (AOS) for far $(\mathrm{F})$ and near $(\mathrm{N})$ fixation and PAT results before surgery, as well as AOS (F) and AOS (N) after surgery and results of binocular function tests were considered. PAT included wearing a prism based on the largest angle for over $60 \mathrm{~min}$.

Results 100 patients (mean age $37 \pm 17$ years) were included in the decEPH group, 82 patients (mean age $30 \pm 13$ years) in the decMET group. For decEPH, before surgery AOS was $25.5 \pm 8.8 \mathrm{pdpt}(\mathrm{F})$ and $23.5 \pm 9.8 \mathrm{pdpt}(\mathrm{N})$. During PAT the AOS increased significantly by $2.7 \pm 4.3$ to $28.2 \pm 8.6 \mathrm{pdpt}(\mathrm{F})$ and by $4.9 \pm 4.5$ to $28.3 \pm 9.5 \mathrm{pdpt}(\mathrm{N})$. Altogether, in $82 \%$ of decEPH patients AOS (F) and/ or AOS (N) inor decreased by at least 3 pdpt. For decMET, before
\end{abstract}

surgery AOS was $28.6 \pm 10.8$ pdpt for far $(\mathrm{F})$ and $30.9 \pm 11.8$ pdpt for near fixation $(\mathrm{N})$. During PAT the AOS increased significantly by $4.2 \pm 5.8$ to $32.5 \pm 9.5 \mathrm{pdpt}(\mathrm{F})$ and by $3.7 \pm 6.1$ to $34.4 \pm 9.5$ pdpt $(\mathrm{N})$. Altogether, in $51 \%$ of decMET patients, AOS (F) and/ or AOS (N) increased by at least $10 \mathrm{pdpt}$, therefore more than $5^{\circ}$ which would have been maximally expected from mictrotropia, or decreased by at least 3 pdpt.

Conclusions The Prism adaptation test (PAT) showed remarkable changes in AOS in both decEPH and decMET. In patients with decEPH, the preoperative assessment of the "true AOS" under PAT reflects a pivotal requirement for successful strabismus surgery, as $82 \%$ had dose relevant angle changes $\geq 3$ pdpt. For patients with decMET the preoperative prism adaptation test is especially of diagnostic value, but also $51 \%$ of decMET patients had changes in AOS beyond the expected microtropic angle ( $\geq 10 \mathrm{pdpt}$ ) or even a dose relevant angle decrease $(\geq 3 \mathrm{pdpt})$.

Keywords Prism adaptation test $\cdot$ Strabismus surgery $\cdot$ Decompensated esophoria $\cdot$ Decompensated microesotropia

C. Gietzelt · J. Fricke · A. Neugebauer .

A. Hedergott $(\bowtie)$

Department of Ophthalmology, Faculty of Medicine and

University Hospital Cologne, University of Cologne,

Kerpener Str. 62, 50937 Cologne, Germany

e-mail: andrea.hedergott@uk-koeln.de 


\section{Introduction}

The Prism adaptation test (PAT) is widely used in Europe as a means to preoperatively evaluate the maximum Angle of squint (AOS) and determine the necessary dosing of strabismus surgery $[1,2]$. Pichler et al. conducted a survey among Austrian strabologic surgeons and evaluated how frequently a PAT was performed prior to strabismus surgery. The results differed remarkably depending on the type of strabismus: While for intermittent exotropia, decompensated exophoria, and decompensated esophoria $90-98 \%$ of surgeons answered to perform PAT prior to strabismus surgery, in patients with infantile esotropia only $49 \%$ stated to perform PAT prior to surgery [1].

Evidence from the literature suggests that for acquired esotropia, so-called augmented surgery, in which the surgery was planned according to the prismadapted angle of squint, leads to significantly better results than surgery which was planned without PAT [3]. However, it remained unclear, whether the increased success was really due to the prism adaptation test or simply due to an increased total amount of surgery [4] or there might have been other bias to the study [5].

One other missing factor in most studies is, that the authors do not differentiate between different types of acquired non-accommodative esotropia [6-8]. Therefore, the aim of this study was to evaluate the effect of PAT on the AOS in decompensated esophoria (decEPH) and decompensated microesotropia (decMET).

The U.S. American literature describes Acute acquired comitant esotropia (AACE) as a disease with sudden onset of large-angle esotropia with preceding intermittent diplopia [9]. Buch et al. [10] found acute accommodative strabismus (31\%) and decompensated esophoria and decompensated monofixation syndrome (together 27\%) to be the most common subtypes of AACE in children (1-15 years old).

Microesotropia (MET), also called fixation disparity or monofixational phoria in the U.S.A., is characterized by a small convergent angle of squint with anomalous retinal correspondence [11]. While sensory fusion may be achieved with subnormal levels of stereopsis, full motor fusion of the small manifest angle is not possible due to anomalous correspondence. Therefore, full motor fusion also cannot be achieved by prisms or surgery. Besides reduced binocular function and a small manifest esotropia revealed by cover-uncover-test, patients often show anisometropia and amblyopia. MET can decompensate into a larger angle (decompensated microesotropia (decMET)), often with diplopia, which can be corrected by prisms or surgery. Postoperatively, MET will remain [12-14].

Decompensated esophoria (decEPH) is characterized by latent esodeviations with normal binocular functions which gradually decompensate into manifest esotropia, most often associated with diplopia.

In a small sample of 26 patients, Savino et al. highlighted the importance of preoperative differentiation between decompensated microtropia (also called Monofixation syndrome (MFS)) and other forms of acquired comitant esotropia with diplopia for an accurate aesthetic and functional prognosis and appropriate surgical treatment plan [15]. Further, Ali et al. recently also drew attention to the diagnosis "decompensated esophoria" as a differential diagnosis in young adults with gradually progressive intermittent, horizontal, binocular diplopia converting to concomitant large angle esotropia [16]. Of the esophoric patients who required surgery, $71 \%$ were undercorrected after surgery. The authors did not report whether prism adaptation was performed preoperatively.

In line with a prospective AACE study of Lyons et al. also in our clinical experience, the most frequent AACE types requiring surgery in older children and young adults are decompensated microesotropia (decMET) and decompensated esophoria (decEPH) [17]. In our opinion, preoperative differentiation between the two entities is of clinical importance, as the two diagnoses have different characteristics which can influence the preoperative orthoptic examination result as well as the outcome after surgery.

The goal of this retrospective study was to evaluate the effect of PAT on the angle of squint in decMET and decEPH.

\section{Material and methods}

\section{Patients}

In this single-center retrospective study, we reviewed the medical records of consecutive patients treated by strabismus surgery due to decEPH or decMET 
between 2003 and 2019 at the center. Inclusion of patient records was stopped after a consecutive 100 cases.

Inclusion criteria for all patients were the preoperative diagnosis of decEPH or decMET, patient age of at least 12 years at the time of surgery, and the documentation of a baseline examination with documented PAT results one to three days before surgery and a follow-up examination one day after surgery. Exclusion criteria were additional neurologic symptoms and previous strabismus surgery. The eye which received the strabismus surgery was considered the study eye.

Patients diagnosed with decEPH before surgery were grouped into one subgroup while patients with the preoperative diagnosis of decMET were grouped into the other subgroup. Additional exclusion criteria for the decEPH group were additional surgery on the oblique muscles or postoperatively diagnosed microtropia.

Diagnostic criteria for decMET were an anamnestic small or even unknown convergent AOS which had converted into a larger angle with or without diplopia [11], reduced binocular functions after prism adaptation, with a persisting small manifest esotropia after prism adaptation, with a predominant leading eye.

Decompensated esophoria (decEPH) was diagnosed if latent esodeviations with normal binocular functions gradually had decompensated into manifest esotropia, usually associated with initially intermittent and gradually persisting diplopia, without amblyopia, often associated with myopia, with good stereovision after prism adaptation and without manifest deviation after prism adaptation, with normal ocular motility, regular saccades, lack of continuous incomitance, and absence of accompanying neurological symptoms (such as saccadic pursuits, dysmetric saccades, downbeat nystagmus, ataxia).

\section{Orthoptic and ophthalmologic examination}

The baseline examination was performed one to three days before surgery. The follow-up examination was performed one day postoperatively. All examinations were conducted by experienced ophthalmologists and orthoptists. The following data have been extracted from the medical records:
- Best-corrected visual acuity (BCVA) of the study eye and fellow eye

- Refraction (in pseudophakic patients, anamnestic refraction before cataract surgery was considered) of the study eye and fellow eye

- Prism diopters of Fresnel prism or prism glasses at baseline, if worn

- Family history of strabismus

- Tests for binocular function (Bagolini striated glasses test, Lang I or II stereo test, Titmus stereo test)

- Measurement of the largest Angle of squint (AOS) by alternating prism and cover test at near $(\operatorname{AOS}(\mathrm{N}))$ and far fixation $(\mathrm{AOS}(\mathrm{F}))$ with the head in primary position before and after PAT and postoperatively

Prism adaptation test

At baseline examination, a Prism adaptation test (PAT) was performed in every patient according to the standard operating procedure of our clinic: Based on the largest angle measured with alternating prism and cover test before PAT, single prisms were mounted on the patient's glasses or Plano eyeglasses and worn for at least one hour. Afterward and while the patient was still wearing the eyeglasses with attached prisms, again the $\operatorname{AOS}(\mathrm{N})$ and $\operatorname{AOS}(\mathrm{F})$ were measured and the tests for binocular function were carried out (at least Bagolini Test for far and near fixation).

Ethics and statistics

According to regional medical regulations on retrospective single-center clinical studies $(\$ 15$ of the Professional code of conduct, General Medical Council for the Northern Rhine in accordance with the General Data Protection Regulation GDPR of the European Union), the Ethics Committee of the University of Cologne decided that further approval was not required for this retrospective analysis. Throughout the whole study, the declaration of Helsinki and applicable national regulations and laws were observed.

Statistical analysis was performed using Excel (Microsoft Excel for Mac, Version 15.29.1, Microsoft, USA) and SPSS (IBM SPSS Statistics, Version 25.0, 
IBM, USA). For metric data, mean and standard deviation were calculated. Means were compared between groups with student's paired t-test for data with normal distribution, otherwise with Wilcoxon test. The threshold for statistical significance was set to $p<0.05$.

\section{Results}

We were able to identify 100 patients who met all inclusion and no exclusion criteria for the decEPH group (2008-2019) and 82 patients, who met all inclusion and no exclusion criteria for the decMET group (2003-2019). Detailed epidemiologic data concerning sex, age, family history for strabismus, eye, BCVA, refraction, and preoperative prisms (Fresnel prism or prism glasses) are summarized in Table 1.

In the decEPH group, there was no statistically significant difference in BCVA between the study eyes and the partner eyes (BCVA of study eyes $=0.07 \pm$ $0.7 \log$ Mar, BCVA of partner eyes $=-0.05 \pm 0.6$ $\operatorname{logMar} ; p=0.064)$. In the decMET, group the mean BCVA of the study eyes was significantly lower than that of the partner eyes (BCVA of study eyes $=0.09$ $\pm 0.21 \log$ Mar, BCVA of partner eyes $=-0.013 \pm$ $0.77 \log$ Mar; $p<0.001$ ).

$76 \%$ of all decEPH patients already had Fresnel prisms or prism glasses at baseline examination (with a mean of $12 \pm 10 \mathrm{pdpt}$ for correction of an esophoric or esotropic angle) while only $23 \%$ of all decMET patients had Fresnel prisms or prism glasses at baseline examination (with a mean of $13.5 \pm 8.9 \mathrm{pdpt})$.

Detailed orthoptic data and main outcome concerning AOS before and after PAT, results of the PAT, postoperative AOS, and binocular functions are summarized in Table 2.

The individual changes of AOS with PAT for far and near fixation per patient and diagnosis are displayed in Fig. 1.

\section{Decompensated esophoria}

For decEPH, before surgery AOS was $25.5 \pm 8.8 \mathrm{pdpt}$ (F) and $23.5 \pm 9.8 \mathrm{pdpt}(N)$. During PAT the AOS increased significantly by $2.7 \pm 4.3$ to $28.2 \pm 8.6$ pdpt (F) and by $4.9 \pm 4.5$ to $28.3 \pm 9.5 \mathrm{pdpt}$ $(N)(p<0.001$ respectively).

Table 1 Detailed data concerning sex, age, family history for strabismus, eye, BCVA, refraction and preoperative prisms (Fresnel prism or prism glasses)

\begin{tabular}{|c|c|c|c|c|}
\hline Diagnosis & \multicolumn{2}{|c|}{$\begin{array}{l}\text { „Esophoria“ } \\
\text { dekEPH }(n=100)\end{array}$} & \multicolumn{2}{|c|}{$\begin{array}{l}\text { „Mikroesotropia“ } \\
\text { dekMET }(n=82)\end{array}$} \\
\hline sex, female:male (\%) & $52: 48$ & & $49: 51$ & \\
\hline age at day of surgery (years) & $37 \pm 17$ & & $30 \pm 13$ & \\
\hline mean $\pm \mathrm{SD}$ (range) & $(12-79)$ & & $(12-59)$ & \\
\hline Family history for strabismus & $27 \%$ & & $42 \%$ & \\
\hline \multirow[t]{2}{*}{ Study eye, right:left (\%) } & $62: 38$ & & $50: 50$ & \\
\hline & Study eyes & Fellow eyes & Study eyes & Fellow eyes \\
\hline BCVA (logMAR) & $0.07 \pm 0.7$ & $-0.05 \pm 0.6$ & $0.09 \pm 0.21$ & $-0.013 \pm 0.77$ \\
\hline mean $\pm \mathrm{SD}$ (range) & $(-0.1$ to 0.2$)$ & $(-0.2$ to 0.2$)$ & $(-0.1$ to 1.3$)$ & $(-0.2$ to 0.2$)$ \\
\hline spherical equivalent (dpt) & $-2.27 \pm 2.75$ & $-2.12 \pm 2.67$ & $-0.55 \pm 3.39$ & $-0.76 \pm 3.31$ \\
\hline mean $\pm \mathrm{SD}$ (range) & $\begin{array}{l}(-11.63 \\
\text { to }+2.75)\end{array}$ & $\begin{array}{l}(-11.63 \\
\text { to }+2.5)\end{array}$ & $\begin{array}{l}(-11.88 \text { to } \\
7.25)\end{array}$ & $\begin{array}{c}(-11.63 \text { to } \\
6.75)\end{array}$ \\
\hline myopia $\geq-0.5 \mathrm{dpt}$ & $56 \%$ & & $44 \%$ & \\
\hline hyperopia $\geq+1.0 \mathrm{dpt}$ & $13 \%$ & & $35 \%$ & \\
\hline anisometropia $\geq 1 \mathrm{dpt}$ & $11 \%$ & & $16 \%$ & \\
\hline $\begin{array}{l}\text { prisms preoperatively (Fresnel prisms or prism } \\
\text { glasses) }\end{array}$ & $76 \%$ & & $23 \%$ & \\
\hline
\end{tabular}


Table 2 Detailed orthoptic data and main outcome concerning AOS before and after PAT, results of the PAT, postoperative AOS and binocular functions

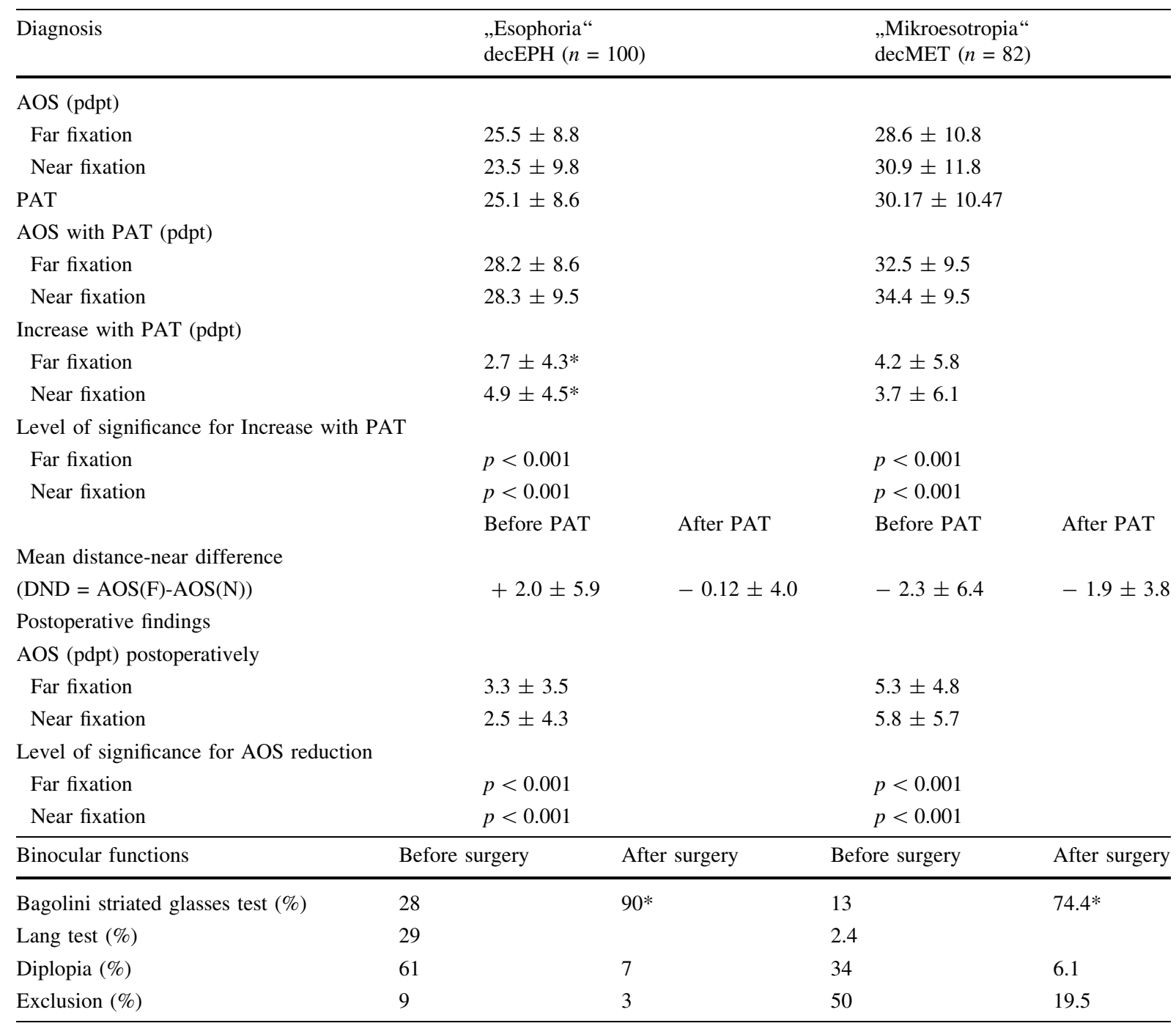

*Statistically significant difference

In our clinical dosage standard, changes in AOS of more than 3 pdpt are considered relevant for dosefinding and change the amount of surgery by $1 \mathrm{~mm}$ recession or plication of the muscle (for patients with refraction due to axial length under $6 \mathrm{D}$ and correction by glasses). We analyzed whether changes with impact on dose-finding occurred after PAT in the decEPH group.

In 52\% of decEPH patients, the AOS (F) enlarged at least by 3 pdpt. $27 \%$ of them had an AOS (F) increase of $\geq 3<6$ pdpt, $18 \%$ an increase of $\geq 6<9$ pdpt, and $7 \%$ had an increase of at least 9 pdpt. In 6\%, AOS (F) was reduced by at least 3 pdpt. The AOS (N) enlarged in $69 \%$ of decEPH patients at least by 3 pdpt. $26 \%$ of them had an AOS $(\mathrm{N})$ increase of $\geq 3$ $<6 \mathrm{pdpt}, 23 \%$ an increase of $\geq 6<9$, and $20 \%$ had an increase of at least 9 pdpt. In $3 \%$, AOS (N) was reduced by at least 3 pdpt. Altogether, in $82 \%$ of decEPH patients AOS $(\mathrm{F})$ and/ or AOS $(\mathrm{N})$ in- or decreased by at least 3 pdpt.

In the decEPH group, the AOS before PAT measured for far fixation was significantly higher than 

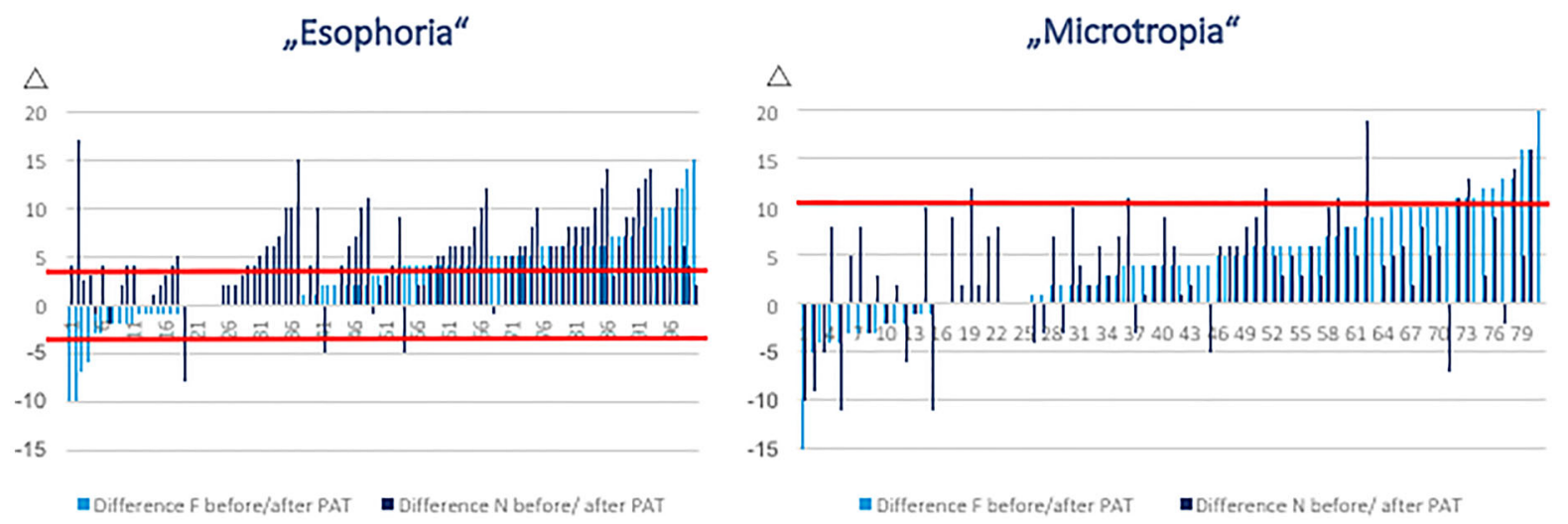

Fig. 1 Individual change of AOS with PAT for far and near fixation for each patient

measured for near fixation ( $\mathrm{p}=0.001)$. The distancenear difference (DND) after PAT decreased significantly compared to the DND before PAT (DND $($ before PAT $)=2.0 \pm 5.9$, DND (after PAT $)=$ $-0.12 \pm 4.0, r=0.54, p<0.001)$.

A subgroup of 76 decEPH patients wore Fresnel prisms or prism glasses preoperatively for several days to weeks (mean prism strength $15.8 \pm 7.9 \mathrm{pdpt}$ ). In most cases, the prisms corrected the AOS only partially: only $32 \%$ of showed a positive Bagolini Test (far fixation) with the prisms. The mean difference between prisms worn preoperatively and PAT was $8.9 \pm 7.8 \mathrm{pdpt}$ (subgroup with positive Bagolini test: $6.8 \pm 5.7 \mathrm{pdpt}$, subgroup with negative Bagolini test or diplopia: $10.1 \pm 8.5 \mathrm{pdpt}$. This difference was not statistically significant $(p=0.134))$. The angle enlargement with PAT compared to AOS before PAT was $2.2 \pm 4.4 \mathrm{pdpt}$ for far distance and $4.2 \pm 4.4 \mathrm{pdpt}$ for near distance. In comparison, the 24 patients without previous Fresnel prisms or prism glasses, the PAT showed a significantly higher angle enlargement both for far and for near distance of $4.3 \pm 3.6 \mathrm{pdpt}$ for far $(p=0.039)$, and $6.7 \pm 4.2 p d p t$ for near distance $(p=0.016)$.

\section{Decompensated microesotropia}

For decMET, before surgery, AOS was $28.6 \pm 10.8$ pdpt for far $(\mathrm{F}), 30.9 \pm 11.8 \mathrm{pdpt}$ for near fixation $(N)$. During PAT the AOS increased significantly by $4.2 \pm 5.8$ to $32.5 \pm 9.5 \mathrm{pdpt}(\mathrm{F})$ and by $3.7 \pm 6.1$ to $34.4 \pm 9.5 \mathrm{pdpt}(N)(p<0.001$ each $)$.

In our clinical dosage standard, enlargement in the AOS of up to $10 \mathrm{pdpt}$ after PAT is to be expected due to the sensory anomaly with anomalous retinal correspondence. However, any further enlargement, or overcorrection by the PAT of more than $3 \mathrm{pdpt}$, would be dose-relevant, see above. We therefore analyzed such changes in the decMET group.

In $21 \%$ of decMET patients, the AOS (F) increased at least by $10 \mathrm{pdpt}$, therefore more than the $5^{\circ}$ which would have been expected from the mictrotropia. $11 \%$ of patients showed an angle reduction of AOS (F) of 3 pdpt and more. The AOS (N) enlarged in $15 \%$ of decMET patients by at least 10 pdpt. $16 \%$ showed an AOS $(\mathrm{N})$ reduction of $3 \mathrm{pdpt}$ and more. Altogether, in $51 \%$ of decMET patients, AOS (F) and/ or AOS (N) increased by at least 10 pdpt or decreased by at least 3 pdpt.

In the decMET group, the AOS measured for near fixation before PAT was significantly higher than the AOS for far fixation. There was no statistically significant difference between the DND before and after PAT (DND (before PAT) $=-2.3 \pm 6.4 \mathrm{pdpt}$, DND $($ after PAT $)=-1.9 \pm 3.8$ pdpt, $r=0.39$, $p=0,455)$.

\section{Comparison of decMET and decEPH groups}

The AOS before surgery was significantly higher in the decMET group compared to the decEPH group both for far and near fixation $(\operatorname{AOS}(F) p=0.023$; $\operatorname{AOS}(\mathrm{N}) p<0.001)$.

Furthermore, the DND was significantly different between the decMET group and the decEPH group $(p<0.001)$ : Before PAT both in the decMET group and the decEPH group there was a significant DND $(p<0.002$ respectively). However, before PAT in the 
decMET group, the mean DND was $-2.3 \pm 6.4$ pdpt, meaning that overall the $\operatorname{AOS}(\mathrm{N})$ was higher than the $\operatorname{AOS}(F)$, the mean preoperative DND in the decEPH group was $+2.0 \pm 5.9 \mathrm{pdpt}$, meaning that overall the $\operatorname{AOS}(\mathrm{F})$ was higher than the $\operatorname{AOS}(\mathrm{N})$. After PAT there was still a significant DND in the decMET group $(p<0.001)$. However, in the decEPH group after PAT there was not any more a significant DND $(p=0.774)$.

In the decMET group, significantly less patients had positive postoperative Bagolini striated glasses test for far fixation than in the decEPH group $(p=0.001)$.

\section{Discussion}

The Prism adaptation test (PAT) is used to find out the "maximum" angle of squint before eye muscle surgery that can be corrected with best binocular outcome to avoid undercorrection [1,3]. When and how long a PAT is performed preoperatively differs from surgeon to surgeon and country to country [1]. Only recently, Zhang et al.[18] compared the therapeutic effects of surgery following prism adaptation test versus surgery alone in Acute acquired comitant esotropia (AACE). They found no significant difference in success rate between the "prism plus surgery" group and "surgery" group 12 months postoperatively. Nevertheless, surgery following prism adaptation test had better outcomes than surgery alone concerning binocular function and recurrence rate.

While many surgeons perform PAT preoperatively in Acute acquired comitant esotropia (AACE), most studies do not distinguish between different underlying causes. Therefore, our study evaluated the effect of Prism adaptation test (PAT) on the Angle of squint (AOS) in two of the main causes of AACE: decompensated esophoria (decEPH) and decompensated microesotropia (decMET).

The diagnosis of decEPH or decMET was made clinically based on multiple clinical characteristics as described above. Those characteristics could therefore also be seen in the statistical analysis of our study population. As expected, BCVA of the eye which received surgery was worse in decMET group compared to decEPH group and furthermore, the difference in BCVA between the study eye and the fellow eye was greater in the decMET group than in the decEPH group. Both binocular vision and stereo function were present in much less of the decMET patients compared to the decEPH patients. Postoperatively the Bagolini test was positive for far distance in $90 \%$ of all decEPH patients, but only $74 \%$ of all decMET patients. It should be noted that the examination was carried out on the first postoperative day.

Our study showed a significant and dose relevant change of the angle of squint with PAT for several decMET and decEPH patients:

In 52\% of decEPH patients the AOS (F) enlarged at least by 3 pdpt, $7 \%$ had an AOS (F) increase of at least 9 pdpt. In only 6\%, AOS (F) was reduced by at least 3 pdpt. Further, AOS (N) enlarged in $69 \%$ of decEPH patients at least by 3 pdpt. Altogether, AOS (F) and/ or AOS $(\mathrm{N})$ in- or decreased by at least 3 pdpt in $82 \%$ of decEPH patients. Angle changes of 3 prisms require a dose adjustment of $1 \mathrm{~mm}$ for eye muscle surgery and are of clinical relevance. Therefore, in patients with decEPH, the PAT is essential for evaluation of the largest angle of squint and correct dose-finding for surgery to avoid undercorrections. This is in line with the existing literature which showed an increase of AOS under PAT in $56-81 \%$ of all patients with acquired esotropia $[2,19]$.

In $21 \%$ of the decMET patients, AOS (F) increased by at least $10 \mathrm{pdpt}$, therefore more than the $5^{\circ}$ which would have been expected from the mictrotropia. $11 \%$ showed an angle reduction of $3 \mathrm{pdpt}$ and more. The AOS (N) enlarged by at least 10 pdpt in $15 \%$ of decMET patients. $16 \%$ showed an AOS $(\mathrm{N})$ reduction of at least 3 pdpt. Altogether, AOS (F) and/ or AOS (N) increased by at least $10 \mathrm{pdpt}$ or decreased by at least 3 pdpt in $51 \%$ of decMET patients. Therefore, the change under PAT is clinically relevant not only for patients with decEPH, but also for almost half of the patients with decMET with need for adjusted dosing of the surgery. In patients with decMET the PAT is also a diagnostic tool: a positive Bagolini test with a persisting small manifest esotropia under PAT strongly supports the diagnosis of microtropia.

Before PAT there was a significant Distance-near difference (DND) in both groups $(p<0.002$ respectively). However, it differed significantly between both groups: while in the decMET patients the $\operatorname{AOS}(\mathrm{F})$ was lower than $(\mathrm{N})$, it was the other way round in decEPH patients. The DND in decMET patients could not be influenced by the PAT. However, in the decEPH group, the PAT lowered the DND significantly, resulting in a no longer significant DND after PAT in the decEPH group. 
In the existing literature, there is only limited information on PAT in patients with DND with larger $\operatorname{AOS}(\mathrm{N})$ than $\operatorname{AOS}(\mathrm{F})$. In one study on PAT in young patients (aged 2-18 years) with esotropia with a DND with larger $\operatorname{AOS}(\mathrm{N})$ than $\operatorname{AOS}(\mathrm{F})$, Kutschke et al. found that augmented surgery on the basis of the enlarged angle under PAT did not lead to a better surgical success rate compared to surgery on the basis of the original near angle [20]. In our decMET group, there also was a DND with $\operatorname{AOS}(N)>\operatorname{AOS}(F)$. However, Kutschke et al. included patients with the preoperative diagnosis of partially accommodative esotropia, acquired esotropia (without further differentiation), and congenital esotropia, so the results cannot be compared fully. Savino et al. compared the sensory status in patients with AACE type I/ II [21] to patients with decompensated monofixation syndrome [15]. Type I AACE (Swan type) is considered due to the interruption of fusion caused by anti-amblyopic occlusion therapy in children without a significant hyperopic refractive error. Type II AACE (BurianFranceschetti type) is characterized by acute onset of concomitant strabismus, often associated with diplopia without an accommodative component even in cases of hyperopia and in the absence of neurological disorders; this esotropia seems to be associated with physical and psychic shock. The patients with monofixation syndrome like our patients showed a larger $\operatorname{AOS}(\mathrm{N})$ than $\operatorname{AOS}(\mathrm{F})$, angle enlargement under PAT, and after further prism increase, almost all patients still had a residual detectable angle of deviation. The abnormal retinal correspondence in patients with decMET causes the angle of squint to further increase as higher and higher prisms are applied during alternating prism and cover test.

In the previous literature there is a very inhomogeneous approach to PAT with time spans for PAT reaching from one hour [22] to 3.5 weeks [3] or even longer [1]. Building up of prisms over weeks before surgery means more visits in the clinic and can often be stressful for patients, especially when they are of young age. Therefore, it seems desirable to shorten the duration of PAT. Altmann et al. examined patients with acquired esotropia under PAT. The angle was built up until it was stable and controlled after $24 \mathrm{~h}$, 4 days, and 7 days. They reported a stable angle in $94 \%$ of the patients in 4- and 7- day visits compared to the 24-h visit. Ela-Dalmann et al. found a similar outcome in patients with acquired esotropia after motor-fusion-test and 1-h prism adaptation compared to the results of the Prism Adaptation Study with several weekly visits to adopt PAT.

While most authors increased the PAT multiple times over a certain time span [23], in our study, only one prism strength was applied for PAT for one hour, and no further increase was performed. However, 76\% of all decEPH patients already wore Fresnel prisms or prism glasses at the baseline examination, respectively $22 \%$ of all decMET patients-not for prism increase though, but for correction of diplopia. Our study showed that the decEPH patients without previous Fresnel prisms or prism glasses showed a significantly higher angle enlargement both for far and for near distance under PAT.

A limitation of our study is that misdiagnosis of decEPH and decMET cannot be avoided completely. Typical difficulties in the diagnosis include young age of patients with unreliable results of orthoptic and stereo function tests [24]. Therefore, to keep the rate of misdiagnosis as low as possible, we excluded patients younger than 12 years of age as well as patients with unreliable orthoptic exam.

Beyond the useful information yielded by PAT and focused on in this study, a prognosis of postoperative binocular functions or the risk of diplopia can be made using PAT.

\section{Conclusions}

The prism adaptation test showed remarkable changes in the orthoptic parameters of patients with decEPH and decMET.

In summary, we conclude that in patients with decEPH the preoperative assessment of the "true AOS" under PAT reflects a pivotal requirement for successful strabismus surgery, as $82 \%$ had an angle inor decrease $\geq 3$ pdpt. For patients with decMET the preoperative prism adaptation test is especially of diagnostic value, but also $51 \%$ of decMET patients had changes in AOS beyond the expected microtropic angle $(\geq 10 \mathrm{pdpt})$ or even a dose relevant angle decrease $(\geq 3 \mathrm{pdpt})$.

Acknowledgements This study was presented at the annual meeting of the German Society of Ophthalmology (DOG) 2020, 09.10. - 11.10., online. 
Author contribution All authors contributed to the study conception and design. Material preparation and data collection and analysis were performed by $\mathrm{CG}, \mathrm{AH}, \mathrm{AN}$, and JF. The first draft of the manuscript was written by $\mathrm{CG}$ and $\mathrm{AH}$ and all authors commented on previous versions of the manuscript. All authors read and approved the final manuscript.

Funding Open Access funding enabled and organized by Projekt DEAL. None.

Data availability The authors have full control of all primary data and they agree to allow International Ophthalmology to review their data upon request.

Code availability via corresponding author.

\section{Declarations}

Conflicts of interest The auhthors declare that they do not have any conflict of interest.

Ethics approval According to regional medical regulations on retrospective single-center clinical studies ( $\$ 15$ of the Professional code of conduct, General Medical Council for the Northern Rhine in accordance with the General Data Protection Regulation GDPR of the European Union), the Ethics Committee of the University of Cologne ruled that approval was not required for this retrospective analysis. Throughout the whole study, the declaration of Helsinki and applicable national regulations and laws were observed.

Open Access This article is licensed under a Creative Commons Attribution 4.0 International License, which permits use, sharing, adaptation, distribution and reproduction in any medium or format, as long as you give appropriate credit to the original author(s) and the source, provide a link to the Creative Commons licence, and indicate if changes were made. The images or other third party material in this article are included in the article's Creative Commons licence, unless indicated otherwise in a credit line to the material. If material is not included in the article's Creative Commons licence and your intended use is not permitted by statutory regulation or exceeds the permitted use, you will need to obtain permission directly from the copyright holder. To view a copy of this licence, visit http://creativecommons.org/licenses/by/4.0/.

\section{References}

1. Pichler U, Rohleder M, Ehrt O (2018) Prism adaptation test before strabismus surgery: Results of a survey and literature review. Ophthalmologe 115(2):123-130. https://doi.org/10. 1007/s00347-017-0457-3

2. Altman M, Baker JD, Petrunak J, Schweers M (1999) Can prism adaptation for acquired esotropia be accomplished in a shorter time frame? J Aapos 3(5):259-262

3. Efficacy of prism adaptation in the surgical management of acquired esotropia. Prism Adaptation Study Research Group (1990). Arch Ophthalmol 108 (9):1248-1256
4. Mims JL 3rd (1991) Wood RC Is prism adaptation helpful for children with acquired esotropia? Arch Ophthalmol 109(4):466-467. https://doi.org/10.1001/archopht.1991. 01080040026010

5. Giangiacomo J (1991) Efficacy of prism adaptation in the surgical management of acquired esotropia. Arch Ophthalmol 109(6):765. https://doi.org/10.1001/archopht.1991. 01080060019002

6. Akbari MR, Mehrabi Bahar MR, Mirmohammadsadeghi A, Bayat R, Masoumi A (2018) Short prism adaptation test in patients with acquired nonaccommodative esotropia; clinical findings and surgical outcome. J AAPOS Official Publication Am Assoc Pediatric Ophthalmol Strabismus 22(5):352-355. https://doi.org/10.1016/j.jaapos.2018.05. 018

7. Jacobs SM, Green-Simms A, Diehl NN, Mohney BG (2011) Long-term follow-up of acquired nonaccommodative esotropia in a population-based cohort. Ophthalmology 118(6):1170-1174. https://doi.org/10.1016/j.ophtha.2010. 10.032

8. Dotan G, Keshet Y, Qureshi HM, Friling R, Yahalom C (2020) When pediatric acute acquired comitant esotropia is not caused by a neurological disease. J AAPOS. https://doi. org/10.1016/j.jaapos.2019.09.016

9. Gilbert AL, Koo EB, Heidary G (2017) Evaluation and Management of Acute Acquired Comitant Esotropia in Children. Seminars Ophthalmol 32(1):8-13. https://doi.org/ 10.1080/08820538.2016.1228398

10. Buch H, Vinding T (2015) Acute acquired comitant esotropia of childhood: a classification based on 48 children. Acta Ophthalmol 93(6):568-574. https://doi.org/10.1111/ aos. 12730

11. Kaufmann HS, H. (2012) Erworbenes Innenschielen und Mikroesotropie. Strabismus, vol 4. Auflage. Thieme Verlag Stuttgart, New York, pp 231-237

12. Lang J (1969) Microtropia. Arch Ophthalmol 81(6):758-762. https://doi.org/10.1001/archopht.1969. 00990010760002

13. Lang J (1974) Management of microtropia. Br J Ophthalmol 58(3):281-292. https://doi.org/10.1136/bjo.58.3.281

14. Sloper J (2017) Special esotropias. In: SR Lambert CL (ed) Taylor \& Hoyt's Pediatric Ophthalmology. Elseviert, pp 806-809

15. Savino G, Abed E, Rebecchi MT, Spreca M, Tredici C, Dickmann A (2016) Acute acquired concomitant esotropia and decompensated monofixation syndrome: a sensorymotor status assessment. Canadian $\mathrm{J}$ Ophthalmol 51(4):258-264. https://doi.org/10.1016/j.jcjo.2016.02.003

16. Ali MH, Berry S, Qureshi A, Rattanalert N, Demer JL (2018) Decompensated Esophoria as a Benign Cause of Acquired Esotropia. Am J Ophthalmol 194:95-100. https:// doi.org/10.1016/j.ajo.2018.07.007

17. Lyons CJ, Tiffin PA, Oystreck D (1999) Acute acquired comitant esotropia: a prospective study. Eye (Lond) $13(\mathrm{Pt}$ 5):617-620. https://doi.org/10.1038/eye.1999.169

18. Zhang P, Zhang Y, Gao L, Yang J (2020) Comparison of the therapeutic effects of surgery following prism adaptation test versus surgery alone in acute acquired comitant esotropia. BMC Ophthalmol 20(1):303. https://doi.org/10. 1186/s12886-020-01574-y 
19. Ohtsuki H, Hasebe S, Tadokoro Y, Kishimoto F, Watanabe S, Okano M (1993) Preoperative prism correction in patients. Graefe's Arch Clin Experimental Ophthalmol 231(2):71-75

20. Kutschke PJ, Keech RV (2001) Surgical outcome after prism adaptation for esotropia with a distance-near disparity. J AAPOS 5(3):189-192. https://doi.org/10.1067/mpa. 2001.115219

21. Burian HM, Miller JE (1958) Comitant convergent strabismus with acute onset. Am J Ophthalmol 45(4 Pt 2):55-64. https://doi.org/10.1016/0002-9394(58)90223-x

22. Ela-Dalman N, Velez G, Thacker N, Britt MT, Velez FG (2006) Maximum motor fusion combined with one-hour preoperative prism adaptation test in patients with acquired esotropia. J AAPOS 10(6):561-564. https://doi.org/10. 1016/j.jaapos.2006.09.011

23. Schildwachter-von Langenthal A, Kommerell G, Klein U, Simonsz HJ (1989) Preoperative prism adaptation test in normosensoric strabismus. Graefe's Arch Clinical Exp Ophthalmol 227(3):206-208. https://doi.org/10.1007/ bf02172749

24. Piantanida AC, Spera M, Nobili R, Gerosa G (2018) Statistics of TNO stereotest for the diagnosis of microesotropia in children. Eur J Ophthalmol 28(3):259-263

Publisher's Note Springer Nature remains neutral with regard to jurisdictional claims in published maps and institutional affiliations. 